\title{
Research Paper \\ Demographics of Pediatric Orbital Lesions: A Tertiary Eye Center Experience in Saudi Arabia
}

\author{
Hind Manaa Alkatan ${ }^{1,2,3,}$, Faisal Al Marek ${ }^{4}$, Sahar Elkhamary ${ }^{5, \dagger}$ \\ ${ }^{1}$ Department of Ophthalmology, College of Medicine, King Saud University, PO Box 18097, Riyadh, Postal code 11415, Saudi Arabia \\ ${ }^{2}$ Department of Pathology, College of Medicine, King Saud University, Riyadh, Saudi Arabia \\ ${ }^{3}$ Department of Pathology and Laboratory Medicine, King Khaled Eye Specialist Hospital, Riyadh, Saudi Arabia \\ ${ }^{4}$ Department of Ophthalmology, Al-Imam Muhammad Ibn Saud Islamic University, Riyadh, Saudi Arabia \\ ${ }^{5}$ Department of Diagnostic Radiology, Mansoura Faculty Of Medicine, Egypt
}

\section{ARTICLE INFO}

Article History

Received 12 March 2016

Accepted 7 November 2017

Keywords

Cyst

neoplasm

orbit

pediatric

vascular

\begin{abstract}
Orbital lesions vary in their classification, incidence, and presentation depending on the age and geographic distribution. Such lesions in the pediatric age group have been studied extensively because of the possibility of faster progression of orbital involvement and the higher risk of morbidity in this age group in which vision is still developing. In Saudi Arabia, orbital lesions were studied over a 6-year period in the late 1980s, when retinoblastoma cases used to present late with orbital involvement. In this study, we revisited the same topic 20 years later aiming to find out the most recent prevalence of orbital lesions in a similar population of patients over a longer period (14 years) in the same eye center, and compare the current results to other reports worldwide. A total of 107 lesions from 106 patients were identified by tissue diagnosis, of which more than half of the lesions were benign cystic (being the most common), vasculogenic, and inflammatory in 63\% [95\% confidence interval (CI, 53.3-72.0)] of all biopsied lesions. Neoplasms accounted for 37\% [95\% CI (28.0-45.8)] with rhabdomyosarcoma being the most common, accounting for about one third of neoplasms, and no orbital cases of retinoblastoma were found. Our results demonstrated different distribution of orbital lesions in recent years reflecting the indirect effect of the improved health awareness and medical care in Saudi Arabia. This baseline demographic study is expected to be helpful for further clinical and prognostic studies with emphasis on pediatric orbital malignant lesions, their clinical presentation, management, and prognosis.
\end{abstract}

(C) 2019 Atlantis Press International B.V.

This is an open access article distributed under the CC BY-NC 4.0 license (http://creativecommons.org/licenses/by-nc/4.0/).

\section{INTRODUCTION}

Orbital pathologic conditions comprise a diverse group of pathology including neoplastic and nonneoplastic lesions. These lesions may cause cosmetic problems, severe ophthalmologic complications with vision loss or eye movement deficit. Eventually, some orbital malignancies can be life-threatening. It may be possible to diagnose the type of orbital tumor based on clinical and radiological findings in relation to the patient's age; however, others require biopsy for various reasons (diagnostic, cosmetic, or optic nerve compression).

Lesions of the extraocular orbit in children differ from those in adults. The younger the child, the more acute the onset is and the faster the progression of orbital involvement, and thus the higher the morbidity in these cases. The pathologic diagnosis and hence the management of rare tumors of the orbit can be challenging. A review of the literature discloses variability in the incidence of childhood orbital tumors; this is related to the nature of each reporting center (pathology centers, pediatric hospitals, etc.) and different geographic areas. Incidence also differs in series derived solely from biopsy material compared with those derived from

*Corresponding author.Emails: hindkatan@yahoo.com; hkatan@ksu.edu.sa

${ }^{\dagger}$ Present address: Department of Diagnostic Radiology, Mansoura Faculty of Medicine, Egypt. clinical diagnosis without histopathological verification. Shields et al. [1] proposed a thorough classification of orbital tumors. A modified classification has been used by our group, which allows easier comparison of one published series with another. This classification is shown in Table 1 . The major reviews include a study from the Wills eye hospital over a 20-year period by Shields et al. [1], which included 250 biopsied tumors. The same group also published another review of 645 biopsies of space-occupying lesions of the orbit, showing the incidence of these lesions with a proposal of a classification [2]. Similarly, Kodsi et al. [3] published a review of 340 histopathologically proven orbital tumors in children. A larger study where analysis of 2480 space-occupying lesions of the orbit was carried out in Naples University by Bonavolontà et al. [4]. However, Bullock et al. [5] reviewed orbital tumors diagnosed clinically and/or histopathologically over a 12-year period.

The prevalence of orbital malignancies in pediatric and adolescents ranges from $8 \%$ to $18 \%[1-3,6]$. Several reports have shown the variability in the prevalence of orbital neoplastic lesions in these age groups in relation to the geographic area. For example, primary malignant tumors of the orbit such as rhabdomyosarcoma account for the majority of orbital malignancies in the United States and Western European studies [1-3,6]. However, in India, secondary tumors were the most common cause of pediatric orbital malignancies as described by 
Table 1 Modified shield's classification of orbital lesions

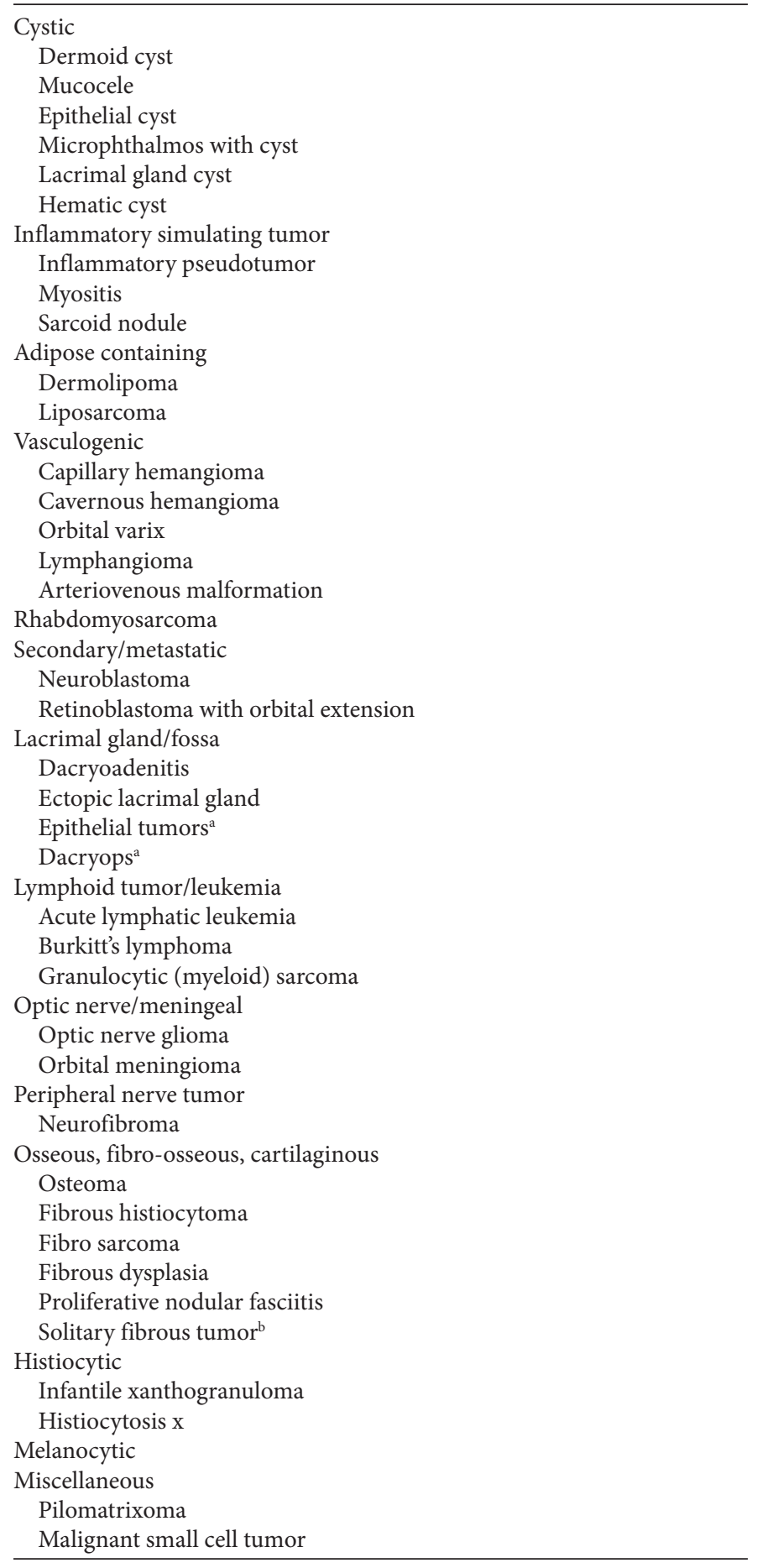

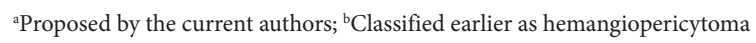
(vasculogenic).

Bakhshi et al. [7]. In Africa Burkitt's lymphoma was the most common neoplastic cause of childhood proptosis [8].

In Saudi Arabia, Johnson et al. [9] reviewed the pediatric orbital tumors in a tertiary eye center and reported that orbital extension of retinoblastoma was the most common lesion followed by vascular lesions, whereas cystic lesions were the third most common. Similar findings were reported by Günalp and Gündüz [10] in Turkey. In this study, we included patients aged $\leq 18$ years who underwent a biopsy for an orbital mass over a 14 -year period in the same center $\sim 20$ years later. We were aiming to report any change or a new trend in the current prevalence of various childhood orbital tumors in comparison with that in a previous study of a similar population in Saudi Arabia.

\section{MATERIALS AND METHODS}

All patients aged $\leq 18$ years or younger who underwent orbital biopsy at King Khaled Eye Specialist Hospital (KKESH) from January 2000 to December 2013 were included. The pathology files listed with the final recorded histopathological diagnosis, which was carried out at KKESH, were reviewed. A single pathologist at the Pathology Department of KKESH reviewed all histopathological slides for confirmation of the final tissue diagnosis. A specially designed data collection sheet (Appendix 1) was used and completed during detailed reviews of the medical records including the demographic information (age, sex, nationality, and city), initial clinical presentation (date of initial presentation, affected side, symptoms, duration of symptoms), medical and surgical history, family history, clinical examination findings, and provisional clinical diagnosis. Lesions confined to the eyelid, conjunctiva, and intraocular lesions were excluded. However, lesions originating from the orbit and extending to involve the eyelid or conjunctiva were included in the study. We also included intraocular lesions with orbital involvement and metastatic lesions to the orbit.

Using a modification of a classification of orbital tumors published earlier by the Shields' study [1], the diagnosis was categorized under one of the major groups of lesions (cystic, vasculogenic, rhabdomyosarcoma, lymphoproliferative, histiocytic, fibrocytic, osseous/ fibro-osseous/cartilaginous, peripheral nerve tumors, optic nerve tumors, infantile/embryonic, secondary, and metastatic). Each major category was given a numeric code with a letter code for the subcategories. The code was used to allow ease of matching the final histopathological diagnosis with the provisional clinical diagnosis.

Descriptive analysis of the data was performed and percentages and 95\% CI reported. Chi-square analysis for the percentage distribution of the 107 classified lesions type in our report versus other comparable large series reported elsewhere and one-way analysis of variance (ANOVA) to test the significance of the duration of symptoms among patients referred from different provinces were conducted.

The study was approved by the Human Ethics Committee/ Institutional Review Board (HEC/IRB) committee at KKESH with the Research Proposal \#RP 1520-R. The research project was granted expedited approval as a retrospective type of study dated February 24,2015 . A general informed consent was obtained by the patients' guardian as part of the common practice at our institution.

\section{RESULTS}

There were 107 biopsied lesions from 106 patients (with one patient showing bilateral histiocytic lesions) involving the orbit during the period encompassed by this study ( 13 years) for patients aged 18 years or younger. The orbital pathological lesions identified included: cystic, vascular, inflammatory, structural lesions, and 40 lesions-in various categories-which were considered true tumor proliferations, either benign or malignant. 
Cystic lesions accounted for 47 cases (43.9\%) [95\% CI (34.6-53.3)], vasculogenic for 17 (15.8\%) [95\% CI (9.3-24.3)], rhabdomyosarcoma for $11(10.2 \%)$ [95\% CI (4.7-15.9)], and peripheral nerve tumors for seven $(6.5 \%)$ [95\% CI (1.9-12.1)]. The distribution of the type of all the tumors and lesions is as shown in Table 2.

The pediatric orbital lesions, which were considered as true tumor proliferations accounted for 37\% [95\% CI (28.0-45.8)] - only of all biopsied lesions. More than half of the lesions were benign cysts (being the most common), vasculogenic, and inflammatory in 63\% [95\% CI (53.3-72.0)] of all the cases, this difference was statistically significant $(p<0.001)$.

The cystic lesions were mostly dermoid cysts in 29/47 followed by developmental cysts associated with microphthalmos in 12/47, then by other less-common types of cysts such as pilar and hematic cysts. The vasculogenic group was mostly lymphangiomas with a single case of capillary hemangioma.

Because the focus of our study is true neoplasm (40/107), which accounted for 37\% [95\% CI (28.0-45.8)] only of all biopsied orbital lesions, the general demographics for these 40 cases were analyzed.

The demographic information regarding age and sex of the total 40 patients is represented in Table 3 , which shows male predominance (male:female ratio 5.4:4).

Of these 40 patients, 38 were Saudis and two were non-Saudis (one case was referred from Kuwait and the other from Yemen). The geographic distribution of the 38 Saudi patients according to the Kingdom of Saudi Arabia provinces was as follows: 13 cases from the central province, 16 from the western province, five from the southern province and four from the eastern province, as shown in Figure 1. A difference in mean duration of symptoms was observed in relation to the place of origin (province). Patients from the central region had

Table 2 Total number of pediatric orbital lesions by type

\begin{tabular}{lc}
\hline Tumor/lesion & No. $(\mathbf{\%})[\mathbf{9 5 \%} \mathbf{C I}](\boldsymbol{n}=\mathbf{1 0 7})$ \\
\hline Cystic & $47(43.9)[34.6-53.3]$ \\
Vasculogenic & $17(15.9)[9.3-24.3]$ \\
Rhabdomyosarcoma & $11(10.3)[4.7-15.9]$ \\
Peripheral nerve tumors & $7(6.5)[1.9-12.1]$ \\
Lymphoid tumors and leukemia & $5(4.7)[0.9-9.3]$ \\
Histiocytic tumors & $4(3.7)[0.9-7.5]$ \\
Fibrocytic tumors & $4(3.7)[0.9-7.5]$ \\
Inflammatory lesions (orbital pseudo tumor) & $3(2.8)[0.0-6.5]$ \\
Osseous/fibro-osseous and cartilaginous tumors & $3(2.8)[0.0-6.5]$ \\
Metastatic tumors & $2(1.9)[0.0-4.7]$ \\
Infantile/embryonal & $2(1.9)[0.0-4.7]$ \\
Meningeal and optic nerve tumors & $1(0.9)[0.0-2.8]$ \\
Epithelioid sarcoma & $1(0.9)[0.0-2.8]$ \\
\hline
\end{tabular}

CI, confidence interval.

Table 3 Demographic information of 40 cases with the tissue diagnosis of a true neoplastic orbital lesion

\begin{tabular}{lc}
\hline Category & No. of patients \\
\hline Females & 17 \\
Males & 23 \\
Mean age of female patients (y) & 4.6 \\
Mean age of male patients (y) & 8 \\
Total & 40 \\
\hline
\end{tabular}

a mean duration of symptoms of 2.5 months, from each of the western and eastern regions had 8 months, whereas the mean duration of symptoms for patients coming from the southern region was 32 months. Statistical testing was carried out by performing ANOVA test for the analysis of these results and it was found that there existed a statistically significant difference in these durations $(p<0.001)$.

The prevalence of the orbital neoplasm in order of the frequency is presented in Figure 2, which was further analyzed in relation to sex and age as summarized in Table 4. All cases were unilateral except one patient with histiocytic tumor, who presented with bilateral orbital involvement.

Clinically, the most common presenting complaints in the neoplastic group were proptosis/eye displacement (25 cases) and periocular/eyelid swelling (25 cases). Restricted ocular motility and diplopia were reported in two cases, reduced vision was reported in one case, whereas pain was not reported as a presenting complaint in any of the cases. Table 5 shows the presenting complaint for each major category (based on the final histopathological diagnosis). The provisional clinical diagnosis accurately matched the final histopathological diagnoses of neoplastic lesions in only $32.5 \%$ of the total 40 lesions.

\section{DISCUSSION}

Earlier reported series of space-occupying orbital lesions in children showed variable results; this could be related to different factors such as the nature and geographic location of the reporting center and source of the material reviewed [11-14].

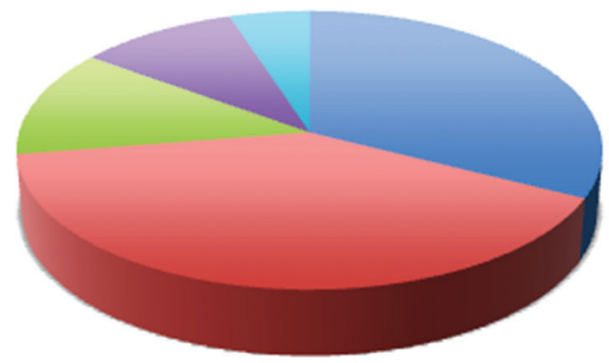

= Central province Westem province Southern province Eastem province Non Saudi

Figure 1 The geographic distribution of the 38 Saudi pediatric patients who were diagnosed with true orbital neoplasm

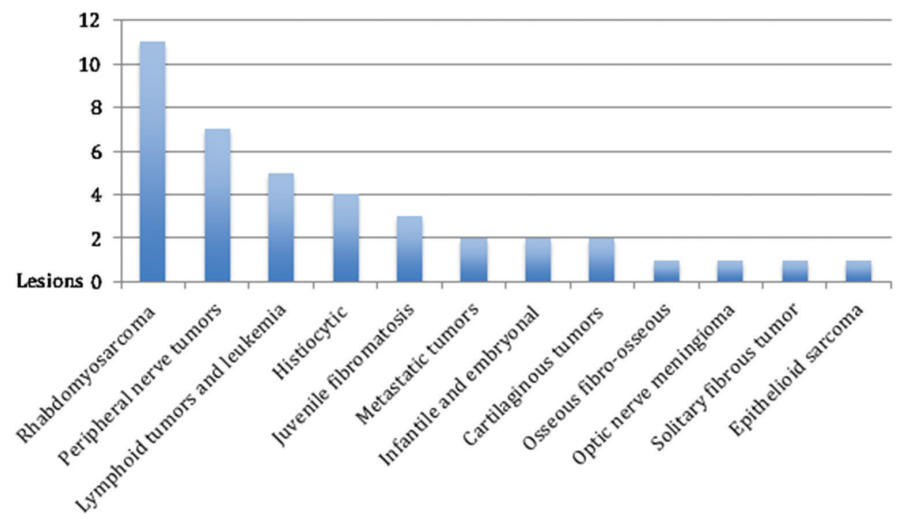

Figure 2 The 40 neoplastic orbital lesions presented in order of frequency according to their final tissue diagnosis 
Table 4 Breakdown of the distribution and demographic information of 40 neoplastic orbital lesions per diagnosis

\begin{tabular}{lcccc}
\hline Category & No. of cases & Percentage (\%) (95\% CI) & Sex (M/F) & Age at presentation $(\mathbf{y})$ \\
\hline Rhabdomyosarcoma & 11 & $27.5(14.0-42.1)$ & $7 / 4$ & Mean 6 \\
Peripheral nerve tumors & 7 & $17.5(5.1-27.8)$ & $3 / 4$ & Mean 4.1 \\
Lymphoid tumors and leukemia & 5 & $12.5(2.7-24.4)$ & $3 / 2$ & Mean 6.6 \\
Histiocytic lesions & 4 & $10(2.3-19.5)$ & $4 / 0$ & Mean 5 \\
Juvenile fibromatosis & 3 & $7.5(2.3-19.6)$ & $1 / 2$ & Mean 4.5 \\
Metastatic tumors & 2 & $5(0.0-12.9)$ & $1 / 1$ & 17 and 2 \\
Infantile and embryonal & 2 & $5(0.0-11.9)$ & $0 / 2$ & 0.4 and 0.9 \\
Cartilaginous tumors & 2 & $5(0.0-12.8)$ & $1 / 1$ & 18 and 2 \\
Osseous fibro-osseous & 1 & $2.5(0.0-8.8)$ & $1 / 0$ & 15 \\
Optic nerve meningioma & 1 & $2.5(0.0-8.5)$ & $1 / 0$ & 13 \\
Solitary fibrous tumor & 1 & $2.5(0.0-8.8)$ & $0 / 1$ & 9 \\
Epithelioid sarcoma & 1 & $2.5(0.0-8.6)$ & $1 / 0$ & 0.2 \\
Total & 40 & 100.0 & &
\end{tabular}

CI, confidence interval; F, female; M, male.

Table 5 Frequency of the clinical symptoms among the 40 neoplastic lesions showing that proptosis and/or the presence of swelling are the most common complaints encountered

\begin{tabular}{lcccccc}
\hline $\begin{array}{l}\text { Category/no. of patients with } \\
\text { presenting complaint }\end{array}$ & No. of cases & $\begin{array}{c}\text { Reduced } \\
\text { vision }\end{array}$ & $\begin{array}{c}\text { Proptosis/eye } \\
\text { displacement }\end{array}$ & Diplopia & $\begin{array}{c}\text { Restricted } \\
\text { ocular motility }\end{array}$ & $\begin{array}{c}\text { Eyelid/periocular } \\
\text { swelling }\end{array}$ \\
\hline Rhabdomyosarcoma & 11 & 0 & 6 & 1 & 1 & 7 \\
Peripheral nerve tumors & 7 & 0 & 5 & 0 & 0 & 6 \\
Lymphoid tumors and leukemia & 5 & 0 & 2 & 0 & 0 & 5 \\
Histiocytic & 4 & 0 & 1 & 0 & 0 & 3 \\
Juvenile fibromatosis & 3 & 1 & 2 & 0 & 1 & 2 \\
Metastatic tumors & 2 & 0 & 2 & 1 & 0 & 0 \\
Infantile and embryonal & 2 & 0 & 2 & 0 & 0 & 0 \\
Cartilaginous tumors & 2 & 0 & 2 & 0 & 0 & 1 \\
Osseous fibro-osseous & 1 & 0 & 0 & 0 & 0 & 0 \\
Optic nerve meningioma & 1 & 0 & 1 & 0 & 0 & 0 \\
Solitary fibrous tumor & 1 & 0 & 1 & 0 & 0 & 0 \\
Epithelioid sarcoma & 1 & 0 & 1 & 0 & 0 & 25 \\
Total & 40 & 1 & 25 & 2 & 2 & \\
\hline
\end{tabular}

Youssefi [15] in histopathological series reported that cystic lesions were the most common (50\%), followed by vascular lesions (14.5\%) in a total of 62 cases over a period of 10 years. Illif and Green [16] reported a series of 174 cases and found that cystic lesions were the most common (34.5\%), followed by vascular lesions (15.5\%).

Bullock et al. [5] reported a clinical series of 141 orbital tumors in children, among which cystic lesions also were the most common $(41.8 \%)$, whether or not a biopsy was performed, followed by vasculogenic lesions (16.3\%). They believed that the frequencies reported in this series were more representative compared with other reports [5]. Shields et al. [1] reviewed 250 consecutive biopsies of orbital masses in children. Cystic lesions were the most common in $41.8 \%$ of the total cases studied, with dermoid cysts being the most common among cystic lesions, and vasculogenic lesions being the second most common (16.3\%), with capillary hemangioma being the most common among the vascular lesions [1]. Templeton [8] reported a series of 60 African cases of orbital tumors in children, wherein they found that Burkitt's lymphoma was the most common of orbital tumors (46.6\%) followed by chloroma (Myeloid sarcoma) (13.3\%). In our series, Burkitt's lymphoma and myeloid sarcoma were not as prevalent as in Africa because the lymphoid group including these two entities constituted only $12.5 \%$ of the neoplastic conditions.

Johnson et al. [9] reported 148 Saudi patients, regardless of whether a biopsy or excision was performed. Secondary neoplasm in a form of orbital retinoblastoma was the most common orbital lesion in $33 \%$ of the cases, followed by vascular lesions in $22.3 \%$ of the cases, and cystic lesions was the third most common in $18.2 \%$ of the cases. The reason why they encountered so many cases of retinoblastoma was not clear to them.

In this series, we encountered 107 cases of orbital masses in children aged $\leq 18$ years in which an orbital excisional or incisional biopsy was performed. More than half of these lesions were nonneoplastic (cystic: being the most common, followed by vasculogenic, and inflammatory) in $63 \%$ cases. Forty cases (37\%) were considered to be true neoplastic lesions.

If we compare our overall results with several similar reports wherein tissue diagnosis has been provided, cystic lesions were the most common tumors (43.9\%) followed by vasculogenic lesions $(15.8 \%)$, and hence our results are consistent with theirs $[1,17,18]$. Because our series involved biopsied orbital lesions, we felt that our results were comparable with those of the Shields' study [1] with the cystic lesions also being the most common orbital pathology in $43.9 \%$ of these children and the dermoid cyst being the most common cystic lesion. However, when comparing the second most common category (vasculogenic) in our series with the Shields' study, we analyzed that lymphangioma is by far the most common with a single case of capillary hemangioma. We believe that clinically, capillary hemangioma would still be the 
most common in our population, but these cases were managed based on their clinical diagnosis without being biopsied, whereas ophthalmologists tend to treat lymphangiomas by excisional biopsy. Our results also match those of Bullock et al. [5] even though their study was based on clinical case series.

Rhabdomyosarcoma is expected to be common when studying orbital neoplasm in children $[17,19,20]$. In our series, it was the third most common lesion involving the orbit (10.2\%) and the most common primary orbital tumor in the neoplastic group $(11 / 40)$ or $27.5 \%$, and more commonly affecting the right side. This is similar to what has been reported by Iliff and Green [16], who too found rhabdomyosarcoma to be the third most common lesion (8.6\%) [16]. Porterfield [19] reported 214 orbital tumors in children; and rhabdomyosarcoma was the most common orbital tumor (26.2\%) with almost an identical result to ours.

However, if we compared the category of neoplastic lesions with an earlier study in our region by Johnson et al. [9] as well as with other studies, in which retinoblastoma was reported as the commonest orbital malignancy; our results were different [5,9,21]. Johnson et al. [9] concluded that secondary orbital retinoblastoma was the most common orbital lesion in 33\% cases in a similar population to ours and in the same particular eye center but within a different era of health awareness and ophthalmic care. These differences in our results compared with Johnson's could be related to the nature and years during which the two studies have been conducted. The first study was carried out from 1983 to 1989, and all cases with orbital lesions were included regardless of whether an orbital biopsy was needed; they reported that many of the cases with secondary orbital retinoblastoma presented with massive proptosis and large fungating tumors in which surgical excision was the main method in managing such cases. They also related the high prevalence of orbital retinoblastoma to late presentation, which most probably has been attributed to low health awareness of the parents. Nowadays, there is better screening for newborns in hospitals, parents tend to seek medical care at earlier stages, and the treatment of retinoblastoma has evolved with use of adjunctive chemotherapy, early management, and less-advanced cases being encountered in our daily ophthalmic practice.

In regard to the geographical distribution, a notable difference in the mean duration of clinical symptoms was observed in relation to the place of origin (province). Patients from the central region had a mean duration of symptoms of 2.5 months, and patients from each of the western and eastern regions had a mean duration of 8 months, whereas the mean duration of symptoms for patients coming from the southern region was 32 months. These differences were statistically significant and could be explained by the nature of the tumors and may not be related to a delay in presentation. However, it is worth studying our health referral system in the kingdom to confirm the relevance of our current observation and its effect on the delay in management of such cases.

Clinically, the most common presenting complaints were eyelid or periocular swelling and proptosis. Family members who observe the abnormalities in the child usually report these findings. The age of the patients could explain why subjective complaints such as reduced vision and diplopia were not reported as frequently as other complaints that were observed by family members. Proptosis, although a relatively rare presenting complaint in childhood, is considered as the hallmark of orbital space-occupying lesions, which can be easily demonstrated by clinical examination [22]. Bakhshi et al. [7] reported a series of 104 cases of malignant childhood proptosis and found that $49.4 \%$ of patients with a malignant orbital mass had clinically documented proptosis, which was unilateral in $86.5 \%$ cases and bilateral in $13.5 \%$ cases. Similarly, Modi et al. [21] reported that proptosis was observed in $49.33 \%$ of orbital tumors in children including both benign and malignant lesions. In our study, proptosis was more frequently evident in $31 / 40$ cases accounting for $77.5 \%$ of cases; wherein all patients had unilateral involvement except for the single case with bilateral orbital involvement. The higher number of proptosis encountered in our study can be explained by late presentation or delayed referral of patients through our health system, especially if they came from rural areas.

There are limitations to our study, it being retrospective in nature within a single institution, wherein we receive cases through a referral system, which is not properly identified or recognized. The clinical diagnosis was not accurately predictive of the proper final tissue diagnosis in most cases. The possible explanation for this is that our study may not represent the routine usual cases that would be encountered by a general ophthalmologist. Some of the simple orbital lesions such as the cystic ones might have been managed locally without referral to KKESH because it is a tertiary care hospital for eye diseases in Saudi Arabia, where more difficult and unusual cases are being referred for further management.

\section{CONCLUSION}

Childhood orbital lesions that require surgical excision are more commonly benign, such as structural lesion or cysts (mostly dermoid cysts) and vascular lesions (mostly lymphangioma). These constituted approximately $60 \%$ of all histopathologically examined orbital lesions in this age group. Neoplastic orbital lesions are less-frequently encountered in $37 \%$ of all cases in our series.

Rhabdomyosarcoma was the most common primary orbital tumor in the neoplastic group accounting for $27.5 \%$ of all neoplasms similar to what has been reported, but the third most common lesion involving the orbit (10.2\%) in total. Secondary retinoblastoma used to be the most common orbital lesion in approximately one third of the cases in our population, but this trend proved to be changed over the past 25 years in our current study, reflecting our advancing techniques in the management of retinoblastoma, thus matching other international eye centers. Also, in contrast to Africa, the lymphocytic/leukemic lesions were much less prevalent. Clinically, proptosis was more frequently evident when compared with other reports, in $77.5 \%$ of all cases, possibly due to relatively late presentation. This late presentation was significantly evident in the cases received from other provinces in the kingdom.

Finally, similar epidemiologic studies on the incidence of tumors in our adult population are recommended, as well as further clinical studies involving the management and outcome for the common orbital neoplastic conditions in different age groups.

\section{CONFLICTS OF INTEREST}

The authors have no conflict of interest in relation to this manuscript. 


\section{REFERENCES}

[1] Shields JA, Bakewell B, Augsburger JJ, Donoso LA, Bernardino V. Space-occupying orbital masses in children. A review of 250 consecutive biopsies. Ophthalmology 1986;93;379-84.

[2] Shields JA, Bakewell B, Augsburger JJ, Flanagan JC. Classification and incidence of space-occupying lesions of the orbit. A survey of 645 biopsies. Arch Ophthalmol 1984;102;1606-11.

[3] Kodsi SR, Shetlar DJ, Campbell RJ, Garrity JA, Bartley GB. A review of 340 orbital tumors in children during a 60 -year period. Am J Ophthalmol 1994;117;177-82.

[4] Bonavolontà G, Strianese D, Grassi P, Comune C, Tranfa F, Uccello $\mathrm{G}$, et al. An analysis of 2,480 space-occupying lesions of the orbit from 1976 to 2011. Ophthal Plast Reconstr Surg 2013;29;79-86.

[5] Bullock JD, Goldberg SH, Rakes SM. Orbital tumors in children. Ophthal Plast Reconstr Surg 1989;5;13-16.

[6] Johansen S, Heegaard S, Bøgeskov L, Prause JU. Orbital spaceoccupying lesions in Denmark 1974-1997. Acta Ophthalmol Scand 2000;78;547-52.

[7] Bakhshi S, Singh P, Chawla N. Malignant childhood proptosis: study of 104 cases. J Pediatr Hematol Oncol 2008;30;73-6.

[8] Templeton AC. Orbital tumours in African children. Br J Ophthalmol 1971;55;254-61.

[9] Johnson TE, Senft SH, Nasr AM, Bergqvist G, Cavender JC. Pediatric orbital tumors in Saudi Arabia. Orbit 1990;9;205-15.

[10] Günalp I, Gündüz K. Pediatric orbital tumors in Turkey. Ophthal Plast Reconstr Surg 1995;11;193-9.

[11] Shields JA, Shields CL, Scartozzi R. Survey of 1264 patients with orbital tumors and simulating lesions: the 2002 Montgomery lecture, part 1. Ophthalmology 2004;111;997-1008.
[12] Bajaj MS, Pushker N, Chaturvedi A, Betharia SM, Kashyap S, Balasubramanya R, et al. Orbital space-occupying lesions in Indian children. J Pediatr Ophthalmol Starbismus 2007;44; 106-11.

[13] Shields JA, Shields CL. Orbital cysts of childhood-classification, clinical features, and management. Surv Ophthalmol 2004;49;281-99.

[14] Ohtsuka K, Hashimoto M, Suzuki Y. A review of 244 orbital tumors in Japanese patients during a 21-year period: origins and locations. Jpn J Ophthalmol 2005;49;49-55.

[15] Youssefi B. Orbital tumors in children: a clinical study of 62 cases. J Pediatr Ophthalmol 1969;6:177-81.

[16] Iliff WJ, Green WR. Orbital tumors in children. In: Jakobiec FA, editor. Ocular and Adnexal Tumors, Birmingham, AL: Aesculapius; 1978, pp. 669-84.

[17] Shields JA, Shields CL. Ocular tumors of childhood. Pediatr Clin North Am 1993;40:805-26.

[18] Chung EM, Smirniotopoulos JG, Specht CS, Schroeder JW, Cube R. From the archives of the AFIP: Pediatric orbit tumors and tumor like lesions: nonosseous lesions of the extraocular orbit. Radiographics 2007;27;1777-99.

[19] Porterfield JF. Orbital tumors in children: A report on 214 cases. Int Ophthalmol Clin 1962;2;319-35.

[20] Castillo BV Jr, Kaufman L. Pediatric tumors of the eye and orbit. Pediatr Clin North Am 2003;50;149-72.

[21] Modi PJ, Shah NA, Bhalodia JN, Gonsai RN. Orbital tumors in children: a descriptive study at tertiary care centre. Natl J Med Res 2013;3;362-6.

[22] Oakhill A, Willshaw H, Mann JR. Unilateral proptosis. Arch Dis Child 1981;56:549-51. 


\section{APPENDIX 1. DATA COLLECTION SHEET}

File \#:

Date of first presentation:

I. Demographic data:

Age: year(s)/month(s)

Date of birth:

Nationality:

II. Clinically:

A. History:

1. Affected eye:

2. Main presenting symptom:

Duration:

- Proptosis

- Globe displacement

- Motility problem

- Pain and tenderness

- Decrease vision

- Diplopia

3. Other complaint

4. Systemic illness

- FH of malignancy

- H/O previous tumor

- $\mathrm{H} / \mathrm{O}$ previous orbital surgery

B. Examination:
Surgical \#:

\begin{tabular}{|c|c|c|c|c|}
\hline $\begin{array}{l}\text { Sex: } \\
\text { Date o }\end{array}$ & $\begin{array}{l}\text { Male } \\
\text { cesenta }\end{array}$ & $\begin{array}{l}2 \text { Fema } \\
\\
\ldots \ldots \ldots . . .\end{array}$ & ............ & \\
\hline 1 Saud & & City: & & \\
\hline 2 Non & udi .... & . & & \\
\hline $1 \mathrm{RT}$ & $2 \mathrm{LT}$ & 3 Both & & \\
\hline 1 Yes & $2 \mathrm{No}$ & $3 \mathrm{Ukn}$ & $\ldots \ldots \ldots \ldots$ wks $/ \mathrm{n}$ & s/yrs \\
\hline 1 Yes & $2 \mathrm{No}$ & $3 \mathrm{Ukn}$ & ............ wks $/ \mathrm{n}$ & s/yrs \\
\hline 1 Yes & $2 \mathrm{No}$ & $3 \mathrm{Ukn}$ & .......... wks $/ \mathrm{n}$ & s/yrs \\
\hline 1 Yes & $2 \mathrm{No}$ & $3 \mathrm{Ukn}$ & ............ wks $/ \mathrm{n}$ & s/yrs \\
\hline 1 Yes & $2 \mathrm{No}$ & $3 \mathrm{Ukn}$ & ............ wks/n & s/yrs \\
\hline 1 Yes & $2 \mathrm{No}$ & $3 \mathrm{Ukn}$ & ............ wks $/ \mathrm{n}$ & s/yrs \\
\hline 1 yes, & cify ... & $\ldots \ldots \ldots$ & $\ldots \ldots \ldots \ldots \ldots \ldots \ldots$ & $2 \mathrm{No}$ \\
\hline 1 yes. & $\ldots \ldots \ldots$ & 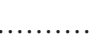 & .................. & $2 \mathrm{No}$ \\
\hline 1 yes & $2 \mathrm{No}$ & & & \\
\hline 1 yes & $2 \mathrm{No}$ & & & \\
\hline $\begin{array}{l}1 \text { yes: } \\
2 \text { No }\end{array}$ & fected & 2 fellow & & \\
\hline
\end{tabular}

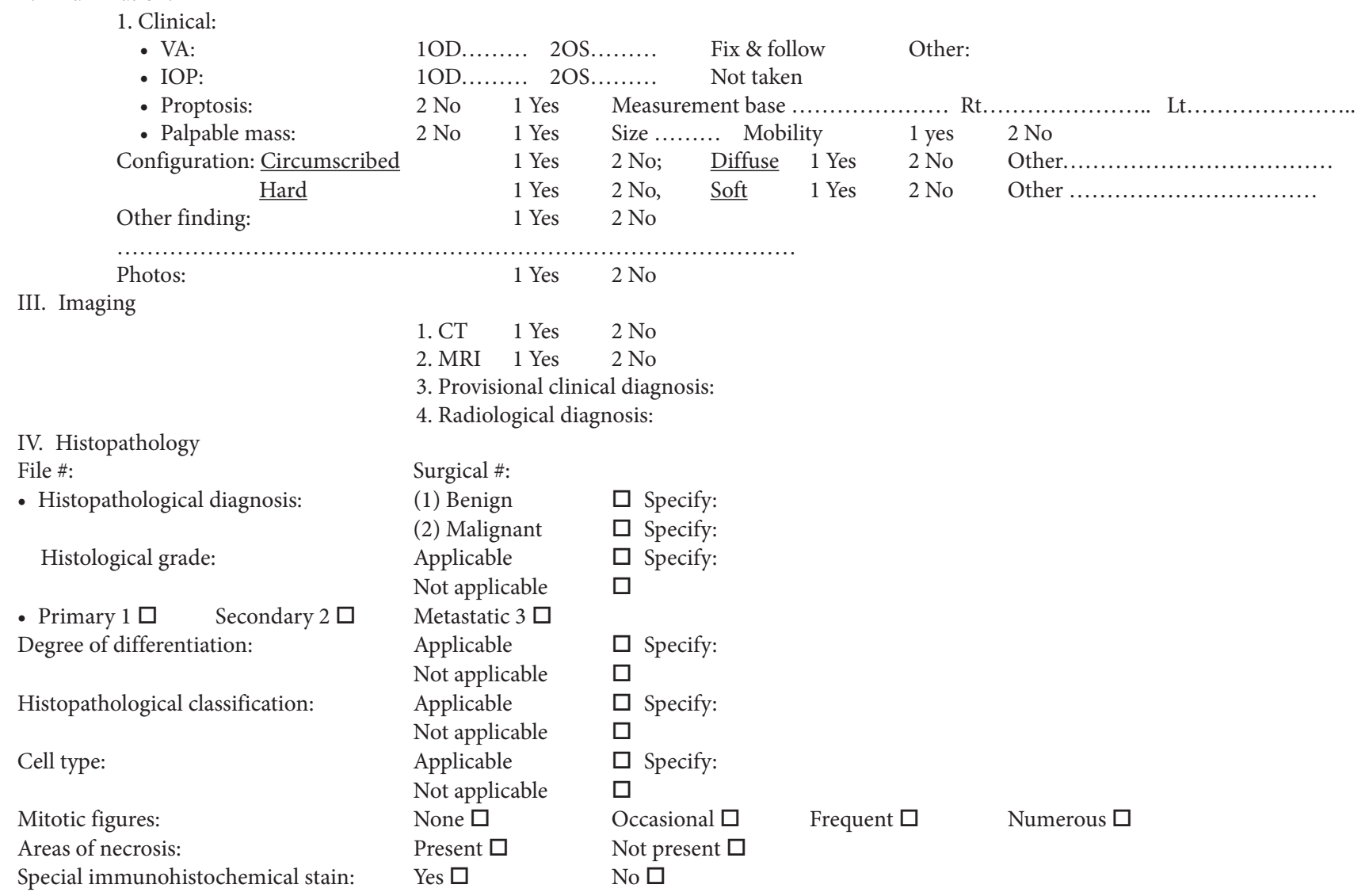




\section{Histologic category:}

Rhabdomyosarcoma (1)

Rhabdomyoma (2)

Embryonal: others (3)

Optic nerve and meningeal: (4)

A. Meningioma

B. Glioma

Peripheral nerve tumors: (5)

A. Neurofibroma

B. Neurilemoma (shwanoma)

C. Amputation neuroma

D. Paragangilioma (chemodectoma)

E. Granular cell myoblastoma

F. Alveolar soft-part sarcoma

Fibrocystic tumors: (6)

A. Fibroma

B. Fibrous histocytoma

C. Fibrosarcoma

D. Nodular fasciitis

E. Juventil fibromatosis

Osseous and fibro-osseous tumors: (7)

A. Osteoma

B. Osteosarcoma

C. Fibrous dysplasia

D. Giant cell tumor

E. Giant cell granuloma

F. Aneurysmal bone cyst

G. Benign osteoblastoma

H. Infantile cortical hyperostosis

I. Ossifying fibroma

J. Brown tumor of hyperparathyroidism

Cartilaginous tumors: (8)

A. Cartilagineous hamartoma

B. Chondroma
C. Chondrosarcoma

D. Mesenchymal Chondrosarcoma

Lipocytic and myxoid tumors: (9)

A. Lipoma

B. Liposarcoma

C. Myxoma

D. Myxoid liposarcoma

Lacrimal gland: (10)

A. Pleomorphic adenoma

B. Pleomorphic adenocarcinoma

C. Adenoid cystic carcinoma

D. Mucoepidermoid carcinoma

E. Benign reactive lymphoid hyperplasia

F. Lymphoma

G. Plasmacytoma

Primary melanocytic tumor: (11)

A. Melanocytic hamartoma

B. Primary orbital melanoma

C. Blue nevus

D. Melanotic progonoma (retinal anlage)

Metastatic tumor to the orbit: (12)

A. Metastatic carcinoma

B. Metastatic carcinoid tumor

C. Neuroblastoma

D. Ewing's sarcoma

Lymphoid tumors and leukemias: (13)

A. Non-Hodgkin's lymphoma

B. Burkitt's lymphoma

C. Lymphobladtic leukemia

D. Benign reactive lymphoid hyperplasia

E. Myelogenous leukemia (sarcoma sarcoma)

Histiocytic. (14)

Juvenile xanthogranuloma (15)

Vasculogenic: (16)
A. Hemangiopericytoma
B. Vascular leiomyoma
C. Vascular leiomyosaroma
D. Hemangiosarcoma
E. Kaposi's sarcoma

Secondary from intraocular origin: (17)
A. Retinoblastoma
B. Uveal melanoma
C. Medulloepithelioma

D. Neurilemoma

Secondary from eyelid origin: (18)
A. Basal cell carcinoma
B. Squamous cell carcinoma
C. Sebaceous carcinoma
D. Melanoma

Secondary from conjunctival origin: (19)

A. Squamous cell carcinoma

B. Melanoma

C. Mucoepidermoid carcinoma

Secondary from Intracranial origin: (20)

A. Astrocytoma

B. Meningioma

Secondary from lacrimal sac origin: (21)
A. Papilloma
B. Melanoma
C. Carcinoma

Para nasal sinus origin: (22)

A. Ethmoid sinus carcinoma

B. Maxillary sinus carcinoma

C. Rhabdomyosarcoma

Nasopharyngeal origin: (23)
A. Carcinoma
B. Melanoma
C. Angiofibroma 